\title{
The Relationship between Environmental Characteristics and Risk Management Practices on Produce Farms: A Systematic Literature Review
}

\author{
Dilhani Nisansala Jayawardhana ${ }^{1}$, Loan Thi Thanh Cao ${ }^{2}$, Thomas A. Yeargin ${ }^{3}$, \\ Kristen E. Gibson ${ }^{3}$ and Angela M. Fraser ${ }^{1, *}$ \\ 1223 Poole Agricultural Center, Department of Food, Nutrition, and Packaging Sciences, Clemson University, \\ Box 340316, Clemson, SC 29634-0316, USA; dilhanj@clemson.edu \\ 2 Research Institute for Biotechnology and Environment, Nong Lam University, \\ Ho Chi Minh City 721400, Vietnam; caoloan@msu.edu \\ 3 Department of Food Science, University of Arkansas System Division of Agriculture, 1371 West Altheimer Dr, \\ Fayetteville, AR 72704, USA; tyeargin@uark.edu (T.A.Y.); keg005@uark.edu (K.E.G.) \\ * Correspondence: afraser@clemson.edu; Tel.: +1-864-656-2652
}

Received: 2 November 2020; Accepted: 20 November 2020; Published: 24 November 2020

check for updates

\begin{abstract}
Produce-associated foodborne disease outbreaks have increased worldwide highlighting the importance of proper implementation of risk management practices (RMP). We determined the relationship between environmental characteristics (i.e., physical resources) of produce farms and implementation of RMP. Following Preferred Reporting Items for Systematic Reviews and Meta-Analyses principles, we identified 36 studies to include in our analysis. Most study data were collected through surveys administered to growers in developed countries. Quality assessment results showed that studies on this topic should be more rigorously conducted (e.g., powering sample sizes and training data collectors) to yield better quality evidence. Agricultural waters were the most common environmental characteristic assessed, with many farms using unsafe water sources. Hygiene aids (e.g., accessible handwashing facilities), were lacking across many farms. Animal intrusion RMP were the least commonly assessed environmental characteristic. Only one study tested the relationship between on-farm environmental characteristics and RMP implementation reporting a positive relationship between accessible handwashing and worker hygiene practices. Grower knowledge and perception of RMP combined with cost and ease in carrying out RMP might influence the availability of physical resources for proper RMP implementation. These results can inform practical interventions aimed to increase adoption of RMP on produce farms.
\end{abstract}

Keywords: physical environment; risk management practices; produce safety; produce farm

\section{Introduction}

Between 2000 and 2013, global per capita annual consumption of fruits and vegetables increased by $25 \%$ [1]. With increased consumption, it is anticipated that the number of produce-associated foodborne disease outbreaks will likely increase since most produce is eaten raw-a known risk factor for foodborne disease transmission [2]. In the United States, over nine million cases of foodborne disease are reported every year with 46\% of cases attributed to produce [3]. Between 1998 and 2013, 972 produce-associated outbreaks were reported in the United States, resulting in 34,674 cases of illness and 72 deaths [4]. During this same period, the percentage of outbreaks attributed to produce also increased from 8\% (1998 to 2001) to 16\% (2010 to 2013) [4]. The European Union (2004 to 2012) and Canada (2008 to 2014) reported similar increases in the number of produce-associated outbreaks [5,6]. 
Contamination of produce can occur at any point along the farm-to-table continuum [7,8]. To our knowledge, no empirical data are available to identify at which point contamination is most common. However, the Food Safety Modernization Act (FSMA) Final Rule on Standards for the Safe Growing, Harvesting, Packing, and Holding of Fruits and Vegetables Grown for Human Consumption (hereafter referred to as the Produce Safety Rule) identifies five sources of contamination in the farm environment: worker health and hygiene, agricultural water, biological soil amendments (BSA) of animal origin, domesticated and wild animals, and equipment, tools and buildings [9]. Risk management practices (RMP) for these contamination sources are addressed in many regional/country-level programs, such as the Global Good Agricultural Practices (GLOBALGAP), prepared by retail members of the Euro-Retailer Produce Working Group; the Guide to Minimize Microbial Food Safety Hazards for Fresh Fruits and Vegetables, prepared by the Food and Drug Administration and U.S. Department of Agriculture; and CanadaGAP [10-12].

To decrease the risk for contamination, training programs aimed to communicate RMP have been prepared by a number of governmental and industry groups. In fact, in the United States, training is now mandated under the Produce Safety Rule for growers earning more than US \$25,000 in annual sales (3-year average, adjusted for inflation) of covered produce (i.e., produce likely to be consumed raw) and those that do not meet the criteria for qualified exemption [13]. To our knowledge, no other country has mandated produce safety training programs for produce growers. The Produce Safety Alliance Grower Training program is the only approved training program in the United States to meet the training requirements of the Produce Safety Rule [14].

The body of evidence demonstrating the effect of training on RMP is still very limited [15]. The conceptual framework for most training programs centers around changing knowledge, with the assumption that practices will improve as knowledge increases [16-20]. Few studies have measured the attitudes and beliefs of growers about RMP or, more importantly, the transfer of knowledge related to the implementation of RMP $[15,19]$.

Many factors can impact the transfer of "training" knowledge in the implementation of RMP. For example, generic training, the most common delivery approach, might not adequately address the unique needs of individual growers as farms vary widely in terms of acreage, commodities grown, production practices, and available resources [21]. Even when growers are adequately trained, a lack of resources may impede the transfer of knowledge into practice. One study involving 1273 local food producers (i.e., farmers, packers, aggregators, and processors) reported that financial resources, time, and facility infrastructure were the three main barriers to implementing RMP [22]. The financial burden of implementing RMP required by the Produce Safety Rule has also been studied [23,24]. However, the relationship between environmental characteristics (i.e., physical resources) of the farm and RMP has not been well studied. We assert the environmental characteristics of a farm are associated with the ability of a grower to implement RMP. The aim of this study was to determine the relationship between environmental characteristics of produce farms and RMP. Our research question was: what environmental characteristics are associated with implementation of RMP? Because there is no standard definition for environmental characteristics, we defined it as the physical resources available on farms, such as toilets and handwashing facilities; equipment, tools, and buildings; types of agricultural water and BSA of animal origin; and farm constructions (e.g., fencing), used to implement RMP during the growing, harvesting, packing, and holding of produce for human consumption. We anticipate our findings can be used to inform food safety interventions.

\section{Methods}

\subsection{Search Strategy}

We followed the Preferred Reporting Items for Systematic Reviews and Meta-Analyses (PRISMA) principles to conduct our search. The six eligibility criteria were: (i) language-English, (ii) publication period-January 2000-August 2019, (iii) geographical area—world, (iv) publication type-peer-reviewed, 
(v) aim —assessment of farm environmental characteristics, and (vi) agriculture type — produce. The eight selected online databases and search engines were: Academic OneFile, Academic Search Complete, Agricola, CAB Abstracts Archives, Food Science and Technology, General Science Full Text, Science Direct, and Web of Science. The search keywords related to on-farm produce safety that were used in the initial search are shown in Table 1.

Table 1. Search keywords used for the systematic literature search.

\begin{tabular}{|c|c|c|c|c|}
\hline Action & & Setting & & Aim \\
\hline Audit OR & & Agriculture OR & & "Produce safety" OR \\
\hline Assessment OR & AND & Produce OR & AND & "Food safety" OR \\
\hline Inspection OR & & Fruits OR & & "Food safety practice" OR \\
\hline Monitoring OR & & Vegetables & & "Good agricultural practice" OR \\
\hline Survey & & & & "Grower practice" \\
\hline
\end{tabular}

The search keyword combination and input methods used were similar when searching all databases. Because Science Direct, a search engine, does not have a similar input option for search words, we entered our search word combination into the advanced search option as follows: "Find articles with these terms-Audit OR Assessment OR Inspection OR Monitoring OR Survey", "title, abstract, or author-specified keywords-(Agriculture OR Produce OR Fruits OR Vegetables) AND ("Produce safety" OR "Food safety" OR "Food safety practice" OR "Good agricultural practice" OR "Grower practice"). We then refined the results by years (2000-2019), article type (research articles), and publication title (Food Control, International Journal of Food Microbiology, Food Policy, Food Microbiology, and Food Research International) to obtain relevant articles. For Web of Science, we used the topic option under basic search to input our search keywords. As Web of Science yielded 25,043 articles during our initial search, we eliminated the irrelevant articles by: (i) excluding the irrelevant databases (Zoological Record, KCI-Korean Journal Database, and Russian Science Citation Index) and (ii) searching "Good agricultural practice OR Grower practice" within the results. The initial search was independently conducted by two researchers.

\subsection{Study Selection}

All articles resulting from the initial search were saved into Legacy RefWorks reference management software (RefWorks LLC, Sparks, NV, USA), and duplicate articles were removed. Then, the title and abstract of each remaining article were screened using the six eligibility criteria (see Search strategy). If the title and abstract met our eligibility criteria, then the full text was reviewed. Studies were eliminated if they used an inappropriate design (e.g., food safety economic assessment, microbiological study alone), were not conducted on a farm (e.g., export market, processing facility), or focused on irrelevant research areas (e.g., consumer perception). Also, we eliminated articles from our analysis if we could not obtain the full text or if it was a conference paper or a review article. The citation listings of all review articles were hand searched to identify additional articles to include in our study sample. Similarly, the citation listings of all articles obtained through full-text screening were hand searched and included in our analysis. Two independent reviewers analyzed all full-text articles and evaluated the quality of the study methods using a checklist that the research team created. Eligible articles were then categorized into direct assessment (DA), indirect assessment (IA), and mixed-method (MM) assessment studies. The environmental characteristics and RMP assessed by studies were categorized into seven assessment domains: (i) worker health and hygiene, (ii) agricultural water, (iii) animal control, (iv) BSA of animal origin, (v) cleaning and sanitization, (vi) harvesting, preparation, and packing, and (vii) storage and transportation to facilitate the analysis. 


\subsection{Quality Assessment}

A quality assessment of the study methods was performed using a checklist created by our research team because, to our knowledge, there is currently no recognized quality assessment checklist for agriculture research. The checklist consisted of eight items categorized into four domains-study design ( 2 items), instrument/tool validation ( 4 items), data collectors ( 1 item), and data ( 1 item) (Table 2$)$. We used different scoring methods for each of our study types-DA, IA, and MM-because of the different data collection methods; thus, the quality criteria that we used cannot be applied to all three study types. The two researchers independently rated each item for each article $(1=$ Yes, $0=$ No or unable to determine, and NA = not applicable), discussed the findings, and reconciled differences between their assessments. The quality criteria used for the quality assessment is not applicable for the outbreak investigation studies, because they were carried out by federal agencies using case-control methods. Therefore, outbreak investigation studies $(n=6)$ were not included in the quality assessment. Table 2 describes the criteria used to score the methods of the articles. All assessments were based on what the authors reported in the paper. Although the authors may have performed the study method in a way which met essential quality criteria, lack of adequate reporting may have led to lower scores for the studies.

Table 2. Criteria used to score methods of the articles.

\begin{tabular}{cc}
\hline Quality Criteria & Criterion Is Met If: \\
\hline Was the sample size appropriate? & $\begin{array}{c}\text { Sample size represents at least } 50 \% \text { of the population } \\
\text { reported by the authors. }\end{array}$ \\
Was the sample recruitment procedure appropriate? & $\begin{array}{c}\text { The authors provided a sufficient description of the } \\
\text { general population, the sample included, and the } \\
\text { sample recruitment procedure so others can repeat } \\
\text { the procedure. }\end{array}$ \\
\hline
\end{tabular}

\begin{tabular}{|c|c|}
\hline \multicolumn{2}{|c|}{ Instrument/tool Validation } \\
\hline Was the protocol reviewed by experts? & $\begin{array}{c}\text { The authors validated the instrument/tool by } \\
\text { expert reviewing. }\end{array}$ \\
\hline $\begin{array}{l}\text { Have any reliability tests been conducted } \\
\text { for the instrument? }\end{array}$ & $\begin{array}{l}\text { The authors validated the instrument/tool by } \\
\text { conducting any reliability test for consistency and } \\
\text { stability in measuring what it is intended to measure. }\end{array}$ \\
\hline $\begin{array}{l}\text { Was the instrument developed by following } \\
\text { an established standard? }\end{array}$ & $\begin{array}{c}\text { The author constructed the instrument/tool based on } \\
\text { established produce safety standards (i.e., Produce } \\
\text { Safety Rule, Global Good Agricultural Practices } \\
\text { (GLOBALGAP)) or previously validated } \\
\text { instrument/tool. }\end{array}$ \\
\hline $\begin{array}{l}\text { Was the instrument pilot tested if the instrument } \\
\text { constructed by the author? }\end{array}$ & $\begin{array}{l}\text { The authors pilot tested the instrument/tool to } \\
\text { identify potential problem areas and deficiencies in } \\
\text { the instrument/tool prior to implementation. }\end{array}$ \\
\hline \multicolumn{2}{|c|}{ Data collectors } \\
\hline Are data collectors trained prior to data collection? & $\begin{array}{l}\text { Data collectors were trained to assure the accuracy } \\
\text { and consistency of the data collection. }\end{array}$ \\
\hline \multicolumn{2}{|c|}{ Data } \\
\hline $\begin{array}{c}\text { Is data cleaned and checked for accuracy } \\
\text { before analysis? }\end{array}$ & The data were checked for accuracy before analysis. \\
\hline
\end{tabular}




\section{Results}

\subsection{Literature Search Strategy and Selection}

The initial search for relevant articles yielded 3083 articles. After removal of duplicates, 2142 articles were identified for possible inclusion in the sample by title and abstract screening. From title and abstract screening, 1740 and 292 irrelevant articles were excluded, respectively. The full texts of the 110 remaining articles were reviewed, and 74 irrelevant articles were removed based on our eligibility criteria-irrelevant study design $(n=24)$, irrelevant research area $(n=26)$, no full text $(n=7)$, conference abstracts $(n=4)$, review articles $(n=5)$, book chapters/reports $(n=2)$, irrelevant settings $(n=4)$, and irrelevant outcomes $(n=2)$. The citation listings of the five review articles were hand searched, and no additional articles were added. The citation listings of the 36 full-text articles were hand searched, resulting in six additional articles. A total of 42 articles were included in our study sample (Figure 1). Of the 42 studies, six studies were outbreak investigation studies [25-30]. Those studies were not included in quality assessment or analysis of study findings. Therefore, a total of 36 studies were used for the final analysis.

\subsection{Study Characteristics}

Eligible articles were assigned to one of three categories based on data collection method [DA $(n=5)$, IA $(n=25)$, and MM $(n=6)$ ], which allowed us to better compare study characteristics (Table 3). The DA category included studies in which data were collected through on-farm visits. Studies in which data were remotely collected using surveys or interviews or on-farms were assigned to the IA category. Studies using both direct and indirect assessment methods were included in the MM category. All eligible studies were published between 2002 and 2018 and were conducted in 12 countries: 21 in the United States, 3 in Brazil, 3 in South Africa, and 1 each in Belgium, Canada, Greece, Lebanon, The Netherlands, Rwanda, Trinidad and Tobago, Turkey, and the United Kingdom. Most studies focused on vegetable-growing environments $(n=32)$ as opposed to fruit growing environments $(n=16)$, and 12 studies focused on both types. The sample size for DA (number of farms) and MM (number of growers) studies ranged from 1 to 166 and 1 to 198, respectively. The sample size (number of growers) for IA studies ranged from 3 to 666. Various methods were used to collect data. For example, DA studies used on-site observations $(n=5)$ and microbial analysis $(n=3)$, whereas IA studies used surveys $(n=20)$, interviews $(n=5)$, and microbial analysis $(n=4)$. MM studies used both DA and IA methods, such as on-site observations, interviews, and microbial analysis, to collect data. 


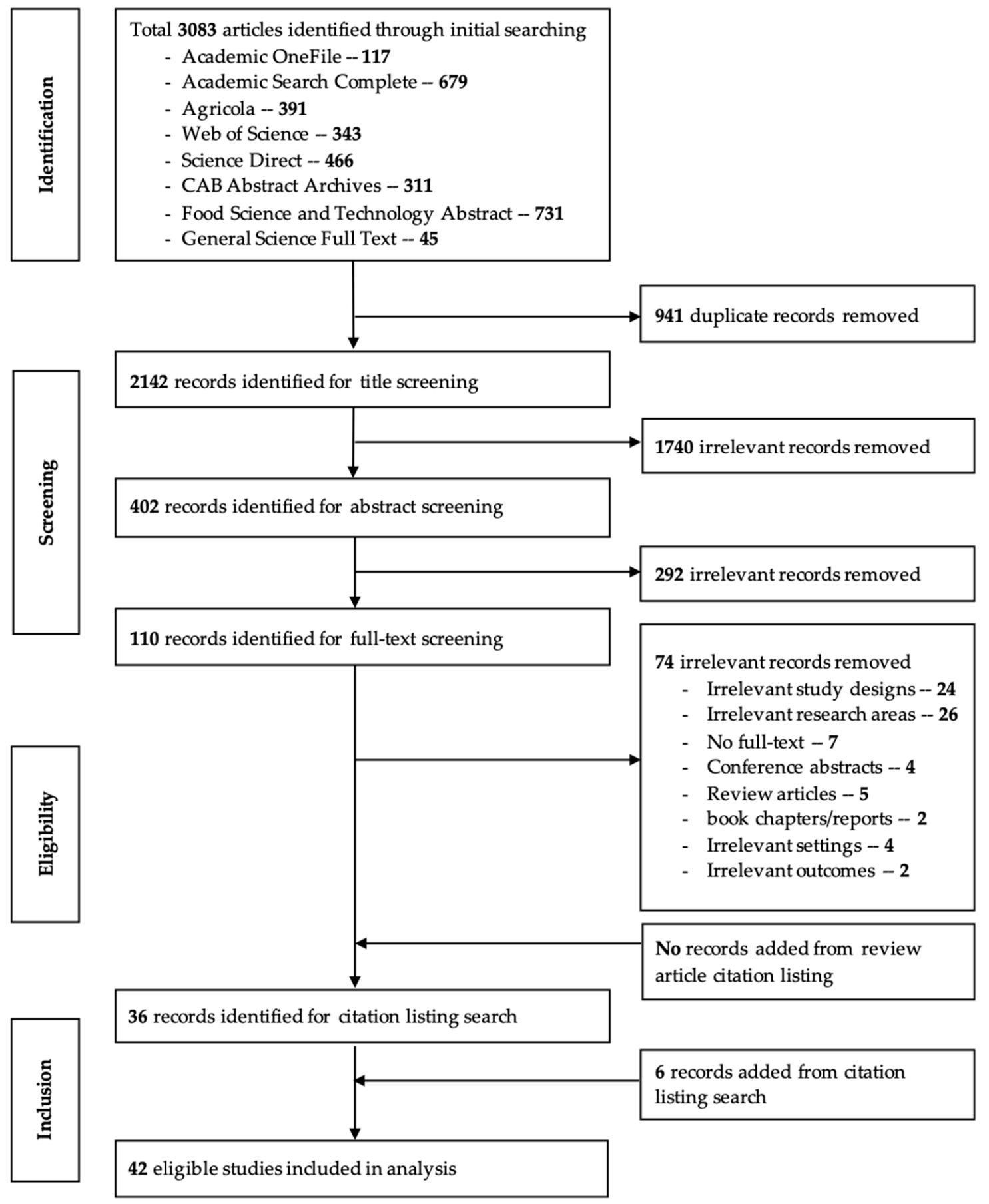

Figure 1. Preferred Reporting Items for Systematic Reviews and Meta-Analyses (PRISMA) flow chart describing the literature search procedure. 
Table 3. Demographics and characteristics of studies included in systematic literature review $(\mathrm{N}=36)$.

\begin{tabular}{|c|c|c|c|c|c|c|c|c|c|}
\hline \multirow[b]{2}{*}{ Study } & \multirow[b]{2}{*}{ Country } & \multicolumn{2}{|c|}{ Produce Type } & \multirow[b]{2}{*}{ Sample Size } & \multicolumn{5}{|c|}{ Methods } \\
\hline & & Fruit & Vegetable & & Observation & Interview & Intervention & Survey & $\begin{array}{c}\text { Microbial } \\
\text { Analysis }\end{array}$ \\
\hline \multicolumn{10}{|l|}{ Direct assessment studies ${ }^{\text {a }}$} \\
\hline Faour-Klingbeil et al., 2016 [31] & Lebanon & & $\mathrm{x}$ & 10 & $\mathrm{x}$ & & & & \\
\hline Luedtke et al., 2003 [32] & Canada & & $\mathrm{x}$ & 166 & $\mathrm{x}$ & & $\mathrm{x}$ & & $\mathrm{x}$ \\
\hline da Cruz et al., 2006 [33] & Brazil & & $\mathrm{x}$ & 1 & $\mathrm{x}$ & & & & $\mathrm{x}$ \\
\hline Pate and Nummer, 2013 [34] & USA & $\mathrm{x}$ & & 6 & $\mathrm{x}$ & & & & \\
\hline Kokkinakis et al., 2007 [35] & Greece & & $x$ & 4 & $\mathrm{x}$ & & & & $\mathrm{x}$ \\
\hline \multicolumn{10}{|l|}{ Indirect assessment studies ${ }^{b}$} \\
\hline Bartz et al., 2015 [36] & Brazil & & $\mathrm{x}$ & 3 & & & & $\mathrm{x}$ & $\mathrm{x}$ \\
\hline Öner and Isin, 2013 [37] & Turkey & $x$ & & 122 & & $\mathrm{x}$ & & & \\
\hline Ganpat et al., 2014 [38] & Trinidad and Tobago & & $\mathrm{x}$ & 196 & & $\mathrm{x}$ & & & \\
\hline Laury-Shaw et al., 2015 [15] & USA & $\mathrm{x}$ & $\mathrm{x}$ & 70 & & & $\mathrm{x}$ & $\mathrm{x}$ & \\
\hline Adalja and Lichtenberg, 2018 [39] & USA & $\mathrm{x}$ & $\mathrm{x}$ & 394 & & & & $\mathrm{x}$ & \\
\hline Van Asseldonk et al., 2018 [40] & Netherlands & & $x$ & 42 & & & $\mathrm{x}$ & $\mathrm{x}$ & \\
\hline Bihn et al., 2013 [41] & USA & $\mathrm{x}$ & $\mathrm{x}$ & 84 & & & & $\mathrm{x}$ & \\
\hline Dzingirayi and Korsten, 2016 [42] & South Africa & & $\mathrm{x}$ & 10 & & $\mathrm{x}$ & $\mathrm{x}$ & & \\
\hline Harrison et al., 2013 [43] & USA & $\mathrm{x}$ & $\mathrm{x}$ & 226 & & & & $\mathrm{x}$ & \\
\hline Ivey et al., 2012 [44] & USA & & $x$ & 210 & & & & $\mathrm{x}$ & \\
\hline Mdluli et al., 2013 [45] & South Africa & & $\mathrm{x}$ & 73 & & $\mathrm{x}$ & & & $\mathrm{x}$ \\
\hline Mukherjee et al., 2007 [46] & USA & $\mathrm{x}$ & $\mathrm{x}$ & 63 & & & & $\mathrm{x}$ & $\mathrm{x}$ \\
\hline Park et al., 2014 [47] & USA & & $\mathrm{x}$ & 12 & & & & $\mathrm{x}$ & \\
\hline Pires et al., 2018 [48] & USA & $\mathrm{x}$ & $\mathrm{x}$ & 666 & & & & $x$ & \\
\hline Soon, 2012 [49] & UK & & $\mathrm{x}$ & 12 & & $\mathrm{x}$ & & & \\
\hline Cohen et al., 2005 [50] & USA & $x$ & & 297 & & & & $\mathrm{x}$ & \\
\hline
\end{tabular}


Table 3. Cont.

\begin{tabular}{|c|c|c|c|c|c|c|c|c|c|}
\hline \multirow[b]{2}{*}{ Study } & \multirow{2}{*}{ Country } & \multicolumn{2}{|c|}{ Produce Type } & \multirow{2}{*}{ Sample Size } & \multicolumn{5}{|c|}{ Methods } \\
\hline & & Fruit & Vegetable & & Observation & Interview & Intervention & Survey & $\begin{array}{c}\text { Microbia } \\
\text { Analysis }\end{array}$ \\
\hline Hultberg et al., 2012 [51] & USA & & $\mathrm{x}$ & 246 & & & & $\mathrm{x}$ & \\
\hline Lichtenberg and Page, 2016 [23] & USA & & $\mathrm{x}$ & 47 & & & & $\mathrm{x}$ & \\
\hline Marine et al., 2016 [52] & USA & $\mathrm{x}$ & $\mathrm{x}$ & 313 & & & $\mathrm{x}$ & $\mathrm{x}$ & \\
\hline Rangarajan et al., 2002 [53] & USA & $\mathrm{x}$ & $\mathrm{x}$ & 213 & & & & $\mathrm{x}$ & \\
\hline Sinkel et al., 2018 [54] & USA & $\mathrm{x}$ & $\mathrm{x}$ & 160 & & & & $\mathrm{x}$ & \\
\hline Tong et al., 2017 [55] & USA & & $\mathrm{x}$ & 39 & & & & $\mathrm{x}$ & $\mathrm{x}$ \\
\hline Jackson et al., 2007 [56] & USA & $\mathrm{x}$ & $\mathrm{x}$ & 596 & & & & $\mathrm{x}$ & \\
\hline Baur et al., 2016 [57] & USA & $\mathrm{x}$ & $\mathrm{x}$ & 588 & & & & $\mathrm{x}$ & \\
\hline Beretti and Stuart, 2008 [58] & USA & & $\mathrm{x}$ & 181 & & & & $\mathrm{x}$ & \\
\hline \multicolumn{10}{|l|}{ Mix method studies ${ }^{c}$} \\
\hline Duvengae and Korsten, 2017 [59] & South Africa & $\mathrm{x}$ & & 1 & $\mathrm{x}$ & $\mathrm{x}$ & & & $\mathrm{x}$ \\
\hline Hamilton et al., 2015 [60] & USA & & $\mathrm{x}$ & 27 & $\mathrm{x}$ & & & $\mathrm{x}$ & \\
\hline Ellis et al., 2005 [61] & USA & $\mathrm{x}$ & $\mathrm{x}$ & 9 & $\mathrm{x}$ & $\mathrm{x}$ & & & \\
\hline Rodrigues et al., 2014 [62] & Brazil & & $\mathrm{x}$ & 3 & $\mathrm{x}$ & $\mathrm{x}$ & & & $\mathrm{x}$ \\
\hline Holvoet et al., 2015 [63] & Belgium & & $\mathrm{x}$ & 8 & & $\mathrm{x}$ & & $\mathrm{x}$ & $\mathrm{x}$ \\
\hline Ssemanda et al., 2018 [64] & Rwanda & & $\mathrm{x}$ & 198 & $\mathrm{x}$ & & & $\mathrm{x}$ & $\mathrm{x}$ \\
\hline
\end{tabular}

$\mathrm{x}$, represents each study's compliance to the measured demographic characteristics. ${ }^{\text {a }}$ Data collectors observed the environmental characteristics or risk management practices and collected the data through on-site visits. ${ }^{b}$ Studies collected the data from produce growers through indirect methods (i.e., survey or interview). ${ }^{c}$ Studies used direct and indirect methods to collect data. 


\subsection{Quality Assessment}

Quality scores ranged from 0 to 3 for DA and MM studies and 0 to 18 for IA studies (Table 4). A common weakness across all studies was that none checked the accuracy of the data before analysis. Further, only one study reported that data collectors were trained before the data collection [60]. Most studies $(n=29)$ did not have an adequate sample size except for two DA studies $[32,33]$ and five IA studies [37,46,49,52,57]. Only two DA studies and three MM studies used appropriate sampling procedures, but most IA studies $(n=18)$ used appropriate sampling procedures. In addition, most DA $(n=3)$, IA $(n=17)$, and MM $(n=6)$ studies validated the assessment instrument/tool using one or more validation processes.

Table 4. Results of the quality assessment of methods of eligible studies $(\mathrm{N}=36)$.

\begin{tabular}{|c|c|c|c|}
\hline \multirow{2}{*}{ Quality Criteria } & \multicolumn{3}{|c|}{$\begin{array}{c}\text { Total Quality Score of Articles Reporting } \\
\text { Each Item }\end{array}$} \\
\hline & $\begin{array}{l}\text { Direct Assessment Studies } \\
\qquad(n=5)\end{array}$ & $\begin{array}{l}\text { In-Direct Assessment Studies } \\
\qquad(n=25)\end{array}$ & $\begin{array}{l}\text { Mixed-Method Studies } \\
(n=6)\end{array}$ \\
\hline \multicolumn{4}{|l|}{ Study Design (2 points) } \\
\hline $\begin{array}{l}\text { Was the sample } \\
\text { size appropriate? }\end{array}$ & 2 & 5 & 0 \\
\hline $\begin{array}{l}\text { Was the sample recruitment } \\
\text { procedure appropriate? }\end{array}$ & 2 & 18 & 3 \\
\hline \multicolumn{4}{|c|}{ Instrument/tool Validation (4 points) } \\
\hline $\begin{array}{l}\text { Was the protocol reviewed } \\
\text { by experts? }\end{array}$ & 0 & 8 & 1 \\
\hline $\begin{array}{l}\text { Have any reliability tests } \\
\text { been conducted for } \\
\text { the instrument? }\end{array}$ & 1 & 3 & 3 \\
\hline $\begin{array}{l}\text { Was the instrument } \\
\text { developed by following an } \\
\text { established standard? }\end{array}$ & 3 & 8 & 3 \\
\hline $\begin{array}{l}\text { Was the instrument pilot } \\
\text { tested if the instrument } \\
\text { constructed by the author? }\end{array}$ & 0 & 9 & 0 \\
\hline \multicolumn{4}{|l|}{ Data Collectors (1 point) } \\
\hline $\begin{array}{l}\text { Are data collectors trained } \\
\text { prior data collection? }\end{array}$ & 0 & $0 *$ & 1 \\
\hline \multicolumn{4}{|l|}{ Data $(1$ point $)$} \\
\hline $\begin{array}{l}\text { Is data cleaned and checked } \\
\text { for accuracy before analysis? }\end{array}$ & 0 & 0 & 0 \\
\hline
\end{tabular}

\subsection{Environmental Characteristics}

Environmental characteristics are presented across the seven assessment domains in Table 5. Among those, the most commonly assessed factor was agricultural water $(n=24)$, followed by worker health and hygiene $(n=20)$, BSA of animal origin $(n=17)$, cleaning and sanitizing $(n=11)$, storage and transportation $(n=11)$, harvesting, preparation, and packing $(n=11)$, and animal control $(n=6)$. The findings associated with harvesting, preparation, and packing, as well as with storage and transportation were not included because the study data were not applicable to our research question. Nearly all studies $(n=35)$ reported the presence/absence or the conditions of the environmental characteristics on farms without determining if there was any statistical relationship between environmental characteristics and RMP. 
Table 5. Comparison of measured outcomes related to environmental characteristics and risk management practices across studies designed to assess the produce farm environment $(\mathrm{N}=36)$.

\begin{tabular}{|c|c|c|c|c|c|c|c|c|c|c|c|c|c|c|c|}
\hline \multirow{2}{*}{\multicolumn{2}{|c|}{ Study }} & \multicolumn{7}{|c|}{ Environmental Characteristics } & \multicolumn{7}{|c|}{ Risk Management Practices } \\
\hline & & $\begin{array}{c}\text { WHH } \\
(n=20)\end{array}$ & $\begin{array}{c}\mathrm{AW} \\
(n=24)\end{array}$ & $\begin{array}{c}\mathrm{AC} \\
(n=6)\end{array}$ & $\begin{array}{l}\text { BSAAO } \\
(n=17)\end{array}$ & $\begin{array}{c}\text { CS } \\
(n=11)\end{array}$ & $\begin{array}{c}\text { HPP } \\
(n=11)\end{array}$ & $\begin{array}{c}\text { ST } \\
(n=11)\end{array}$ & $\begin{array}{c}\text { WHH } \\
(n=19)\end{array}$ & $\begin{array}{c}\mathrm{AW} \\
(n=29)\end{array}$ & $\begin{array}{c}\text { AC } \\
(n=18)\end{array}$ & $\begin{array}{l}\text { BSAAO } \\
(n=27)\end{array}$ & $\begin{array}{c}\text { CS } \\
(n=20)\end{array}$ & $\begin{array}{c}\text { HPP } \\
(n=19)\end{array}$ & $\begin{array}{c}\text { ST } \\
(n=14)\end{array}$ \\
\hline \multicolumn{16}{|c|}{ Direct assessment studies ${ }^{a}$} \\
\hline 1 & Faour-Klingbeil et al., 2016 [31] & & $\mathrm{x}$ & & $\mathrm{x}$ & & & & $\mathrm{x}$ & & $\mathrm{x}$ & $\mathrm{x}$ & & & \\
\hline 2 & Luedtke et al., 2003 [32] & & $\mathrm{x}$ & & & & & & & $\mathrm{x}$ & & & $\mathrm{x}$ & $\mathrm{x}$ & \\
\hline 3 & da Cruz et al., 2006 [33] & $\mathrm{x}$ & $\mathrm{x}$ & $\mathrm{x}$ & & $x$ & $x$ & & $x$ & $\mathrm{x}$ & & $x$ & $\mathrm{x}$ & & $x$ \\
\hline 4 & Pate and Nummer, 2013 [34] & $\mathrm{x}$ & & & & & & & $\mathrm{x}$ & & & & & $\mathrm{x}$ & \\
\hline 5 & Kokkinakis et al., 2007 [35] & & $\mathrm{x}$ & & & & $x^{*}$ & $x^{*}$ & $\mathrm{x}$ & $\mathrm{x}$ & & $\mathrm{x}$ & $\mathrm{x}$ & $\mathrm{x}$ & $\mathrm{x}$ \\
\hline \multicolumn{16}{|c|}{ Indirect assessment studies } \\
\hline 6 & Bartz et al., 2015 [36] & $\mathrm{x}$ & $\mathrm{x}$ & & $\mathrm{x}$ & $\mathrm{x}$ & $\mathrm{x}$ & $\mathrm{x}$ & $\mathrm{x}$ & $\mathrm{x}$ & $\mathrm{x}$ & $\mathrm{x}$ & & & \\
\hline 7 & Öner and Isin, 2013 [37] & $\mathrm{x}$ & & & & & & $x^{*}$ & & & & $\mathrm{x}$ & & & $*$ \\
\hline 8 & Ganpat et al., 2014 [38] & $\mathrm{x}$ & $x$ & & $\mathrm{x}$ & & & $\mathrm{x}$ & $\mathrm{x}$ & $\mathrm{x}$ & $\mathrm{x}$ & $\mathrm{x}$ & $\mathrm{x}$ & $x$ & $\mathrm{x}$ \\
\hline 9 & Laury-Shaw et al., 2015 a [15] & $x$ & & & & & & & $x$ & $x$ & $x$ & & & & \\
\hline 10 & Adalja and Lichtenberg, 2018 [39] & $\mathrm{x}$ & & & & & $\mathrm{x}$ & & & $\mathrm{x}$ & $\mathrm{x}$ & $\mathrm{x}$ & $\mathrm{x}$ & $\mathrm{x}$ & \\
\hline 11 & Van Asseldonk et al., 2018 [40] & & $\mathrm{x}$ & & & & & & & $x$ & & & & & \\
\hline 12 & Bihn et al., 2013 [41] & & $\mathrm{x}$ & & & & & & & $\mathrm{x}$ & & & & & \\
\hline 13 & Dzingirayi and Korsten, 2016 [42] & $x^{*}$ & $x^{*}$ & & & $x^{*}$ & $x^{*}$ & $x^{*}$ & & $x^{*}$ & & & $x^{*}$ & $x^{*}$ & $x^{*}$ \\
\hline 14 & Harrison et al., 2013 [43] & $\mathrm{x}$ & $\mathrm{x}$ & & $\mathrm{x}$ & $\mathrm{x}$ & & $\mathrm{x}$ & & $\mathrm{x}$ & $\mathrm{x}$ & $\mathrm{x}$ & $\mathrm{x}$ & $\mathrm{x}$ & $\mathrm{x}$ \\
\hline 15 & Ivey et al., 2012 [44] & & & & $\mathrm{x}$ & & & & & $\mathrm{x}$ & & $\mathrm{x}$ & $\mathrm{x}$ & & \\
\hline 16 & Mdluli et al., 2013 [45] & & $\mathrm{x}$ & & $\mathrm{x}$ & & & & $\mathrm{x}$ & $\mathrm{x}$ & & $\mathrm{x}$ & $\mathrm{x}$ & & \\
\hline 17 & Mukherjee et al., 2007 [46] & & & & $\mathrm{x}$ & & & & & & & $\mathrm{x}$ & & & \\
\hline 18 & Park et al., 2014 [47] & & & $\mathrm{x}$ & & & & & $\mathrm{x}$ & & $\mathrm{x}$ & $\mathrm{x}$ & & & \\
\hline 19 & Pires et al., 2018 [48] & & & & $x$ & & & & & & & $\mathrm{x}$ & & & \\
\hline 20 & Soon, 2012 [49] & $\mathrm{x}$ & $\mathrm{x}$ & $\mathrm{x}$ & $x$ & $\mathrm{x}$ & $\mathrm{x}$ & $\mathrm{x}$ & $\mathrm{x}$ & $\mathrm{x}$ & $\mathrm{x}$ & $\mathrm{x}$ & $x$ & $\mathrm{x}$ & $\mathrm{x}$ \\
\hline 21 & Cohen et al., 2005 [50] & $\mathrm{x}$ & $\mathrm{x}$ & & $\mathrm{x}$ & $\mathrm{x}$ & & & $\mathrm{x}$ & $\mathrm{x}$ & $\mathrm{x}$ & $\mathrm{x}$ & $\mathrm{x}$ & $\mathrm{x}$ & \\
\hline 22 & Hultberg et al., 2012 [51] & $\mathrm{x}$ & $\mathrm{x}$ & & & & & & $\mathrm{x}$ & $\mathrm{x}$ & $\mathrm{x}$ & $\mathrm{x}$ & $\mathrm{x}$ & $\mathrm{x}$ & $\mathrm{x}$ \\
\hline 23 & Lichtenberg and Page, 2016 [23] & $x^{*}$ & & & & & & & $x^{*}$ & $\mathrm{x}$ & $\mathrm{x}$ & $\mathrm{x}$ & $\mathrm{x}$ & $\mathrm{x}$ & \\
\hline 24 & Marine et al., 2016 [52] & & $x$ & & & & $x$ & & & $\mathrm{x}$ & $\mathrm{x}$ & $\mathrm{x}$ & $\mathrm{x}$ & $\mathrm{x}$ & \\
\hline
\end{tabular}


Table 5. Cont.

\begin{tabular}{|c|c|c|c|c|c|c|c|c|c|c|c|c|c|c|c|}
\hline \multirow{2}{*}{\multicolumn{2}{|c|}{ Study }} & \multicolumn{7}{|c|}{ Environmental Characteristics } & \multicolumn{7}{|c|}{ Risk Management Practices } \\
\hline & & \multirow[t]{2}{*}{$\begin{array}{c}\text { WHH } \\
(n=20)\end{array}$} & \multirow{2}{*}{$\begin{array}{c}\begin{array}{c}\mathrm{AW} \\
(n=24)\end{array} \\
\mathrm{x}\end{array}$} & \multirow[t]{2}{*}{$\begin{array}{c}\mathrm{AC} \\
(n=6)\end{array}$} & \multirow{2}{*}{$\begin{array}{c}\begin{array}{c}\text { BSAAO } \\
(n=17)\end{array} \\
\mathrm{x}\end{array}$} & \multirow[t]{2}{*}{$\begin{array}{c}\text { CS } \\
(n=11)\end{array}$} & \multirow[t]{2}{*}{$\begin{array}{c}\text { HPP } \\
(n=11)\end{array}$} & $\begin{array}{c}\text { ST } \\
(n=11)\end{array}$ & $\begin{array}{c}\text { WHH } \\
(n=19)\end{array}$ & $\begin{array}{c}\mathrm{AW} \\
(n=29)\end{array}$ & \multirow[t]{2}{*}{$\begin{array}{c}\text { AC } \\
(n=18)\end{array}$} & \multirow{2}{*}{$\begin{array}{c}\begin{array}{c}\text { BSAAO } \\
(n=27)\end{array} \\
x\end{array}$} & \multirow[t]{2}{*}{$\begin{array}{c}\text { CS } \\
(n=20)\end{array}$} & \multirow{2}{*}{$\begin{array}{c}\begin{array}{c}\text { HPP } \\
(n=19)\end{array} \\
\mathrm{x}\end{array}$} & $\begin{array}{c}\text { ST } \\
(n=14)\end{array}$ \\
\hline 25 & Rangarajan et al., 2002 [53] & & & & & & & & & $\mathrm{x}$ & & & & & \\
\hline 26 & Sinkel et al., 2018 [54] & $\mathrm{x}$ & $\mathrm{x}$ & & & $x^{*}$ & & $x^{*}$ & $\mathrm{x}$ & $\mathrm{x}$ & & $\mathrm{x}$ & $x^{*}$ & $\mathrm{x}$ & $x^{*}$ \\
\hline 27 & Tong et al., 2017 [55] & & $\mathrm{x}$ & & $\mathrm{x}$ & & $\mathrm{x}$ & & & $\mathrm{x}$ & & $\mathrm{x}$ & & $\mathrm{x}$ & $\mathrm{x}$ \\
\hline 28 & Jackson et al., 2007 [56] & $x^{*}$ & & & & & & & $x^{*}$ & & & & & & \\
\hline 29 & Baur et al., 2016 [57] & & & $\mathrm{x}$ & $\mathrm{x}$ & & & & & $\mathrm{x}$ & $\mathrm{x}$ & $\mathrm{x}$ & & & \\
\hline 30 & Beretti and Stuart, 2008 [58] & & & $\mathrm{x}$ & & & & & & $\mathrm{x}$ & $\mathrm{x}$ & & & & \\
\hline \multicolumn{16}{|c|}{ Mixed method studies } \\
\hline 31 & Hamilton et al., 2015 [60] & $\mathrm{x}$ & $\mathrm{x}$ & & $\mathrm{x}$ & & & & $\mathrm{x}$ & $\mathrm{x}$ & $\mathrm{x}$ & $\mathrm{x}$ & $\mathrm{x}$ & $\mathrm{x}$ & $\mathrm{x}$ \\
\hline 32 & Duvengae and Korsten, 2017 [59] & $\mathrm{x}$ & $\mathrm{x}$ & & & $\mathrm{x}$ & & & & $\mathrm{x}$ & $\mathrm{x}$ & & $\mathrm{x}$ & & \\
\hline 33 & Ellis et al., 2005 [61] & $\mathrm{x}$ & $\mathrm{x}$ & & $\mathrm{x}$ & $\mathrm{x}$ & $\mathrm{x}$ & $\mathrm{x}$ & $\mathrm{x}$ & $\mathrm{x}$ & & $\mathrm{x}$ & $\mathrm{x}$ & $\mathrm{x}$ & $\mathrm{x}$ \\
\hline 34 & Rodrigues et al., 2014 [62] & $x^{*}$ & $x^{*}$ & & $x^{*}$ & $x^{*}$ & $x^{*}$ & $x^{*}$ & $x^{*}$ & $x^{*}$ & & $x^{*}$ & $x^{*}$ & $x^{*}$ & $x^{*}$ \\
\hline 35 & Holvoet et al., 2015 [63] & $x^{*}$ & $\mathrm{x}$ & & $\mathrm{x}$ & $\mathrm{x}$ & $x^{*}$ & $x^{*}$ & $x^{*}$ & $\mathrm{x}$ & $\mathrm{x}$ & $\mathrm{x}$ & $x^{*}$ & $x^{*}$ & $x^{*}$ \\
\hline 36 & Ssemanda et al., 2018 [64] & & $x^{*}$ & $x^{*}$ & & & & & & $x^{*}$ & $x^{*}$ & $\mathrm{x}$ & & & \\
\hline
\end{tabular}


Studies that assessed agricultural water $(n=24)$ mainly focused on water source $(n=20)$ and/or water treatment methods $(n=4)$. For example, Soon (2012) reported that 9 of 12 farms in their study did not treat surface water and well water prior to irrigation [49], but most farms ( $75 \%$ of 12 farms) tested their irrigation water at least annually. On one peach farm, Duvenage and Korsten (2017) reported the farm used untreated or untested water, reportedly mitigating the risk of microbial contamination by using drip irrigation systems [59]. More than half of New York growers (57\% of 84 growers) used surface water for irrigation, with $85 \%$ using overhead irrigation, and did not report other irrigation water sources or irrigation methods [41].

Sanitary facilities associated with worker health and hygiene (e.g., available toilets and handwashing stations) were reported in 13 studies. Of these, nine reported that toilets or handwashing stations were accessible to workers, but only four reported that the toilets on most farms $(>50 \%)$ were within the $\frac{1}{4}$ mile distance required by Occupational Safety and Health Administration (OSHA) [65] or were located near fields $[15,34,36,43]$. Of the six studies that assessed the presence of handwashing aids (e.g., clean water, soap, and paper towels), four reported that more than $50 \%$ of farms had handwashing aids required for proper handwashing $[15,43,50,54]$ with only one reporting less than half $(41 \%)$ of the 27 farms in their sample as having proper handwashing aids [60].

BSA of animal origin were reported in 17 studies, with nearly all $(n=15)$ assessing the type of BSA of animal origin used by growers. According to a study by Pires and colleagues (2018), 58.3\% of 356 U.S. growers who were surveyed reported using raw manure, and over $90.7 \%$ respondents who used raw manure indicated using the 90-120-day waiting period between application and harvest [48]. Furthermore, these same authors assessed storage of BSA of animal origin and reported that only $13.6 \%$ of 309 U.S. growers stored raw or untreated manure in containers or indoor facilities, whereas $76.9 \%$ stored in piles, and $15.9 \%$ used storage pits [48]. In only one study was personal protective equipment associated with manure handling assessed [38]. However, these investigators did not report what personal protective equipment was available or the relationship between personal protective equipment and manure handling practices [38].

The characteristics of cleaning and sanitization of equipment and tools were reported in 11 studies. The following cleaning agents were reported in four studies: soap, vinegar, mild detergent, bleach solution, sulfur/citric solution, ammonia, steam, and/or water [43,49,50,61]. Inadequate sanitizing practices for equipment and tools after cleaning were also reported in four studies $[32,33,43,61]$. According to Cohen and colleagues (2005), only $28 \%$ of 127 farms used bleach or a sanitizing solution, and most used soap with water (35\%) or water alone (37\%) [50]. In addition, less than $40 \%$ of farms sanitized the equipment, tools, and/or food-contact surfaces [43,49,61]. It was also reported that $33 \%$ of 44 farms used the same equipment to haul crops as well as handle garbage and waste [49]. Five study authors reported that equipment and tools were hygienically designed.

Animal control measures were assessed in six studies. In those studies, fences were commonly used $[33,49,57,58]$ with additional control measures reported such as falconers, owl boxes, non-poison traps, poison baits, and copper sulfate [57,58]. Laury-Shaw et al. (2015) reported only $24 \%$ of 41 U.S. growers controlled wild animal contact through fencing or other methods [15]. Furthermore, six study authors reported that wild and/or domestic animals have access to production fields as well as water sources $[31,36,43,52,59,62]$.

\subsection{Relationship between Environmental Characteristics and Risk Management Practices (RMP)}

Only one study performed statistical data analysis to determine the relationship between environmental characteristics and RMP [34]. The remaining studies simply reported the presence/ absence or condition of environmental characteristics. Pate and Nummer (2013) reported a significant difference in the frequency of handwashing among farmworkers who received adequate handwashing aids than those who did not receive adequate handwashing aids [34]. However, four studies did determine the relationship between compliance with good agricultural practices (GAP) standards and implementation of RMP. Of those, three reported that GAP-certified farms practiced RMP more 
frequently than non-certified farms [35,37,38]. In addition, growers with knowledge of GAPs were reported to more likely provide handwashing and toilet facilities for their workers as compared to growers without knowledge about GAPs [56].

\subsection{Relationship between RMP and Presence of Microorganisms}

Of the 36 studies, 11 included a microbiological analysis as a part of their study method (Table 6). Of those, only two studies determined the relationship between RMP and the presence of microorganisms [46,63]. In these two studies, use of animal waste in fertilization of fresh fruits and vegetables significantly increased the risk of Escherichia coli in fresh produce grown in semi-organic and organic farms [46]. Further, Mukherjee et al. (2007) reported that the aging of non-composted manure for more than six months contributed significantly to reducing the risk of E. coli [46]. According to both studies, the risk of E. coli was significantly higher at farms that used cattle manure than farms that used poultry, hog, and/or horse manure or treated organic fertilizer [46,63]. In addition, Holvoet et al. (2015) analyzed the prevalence of microorganisms in borehole and open well water and found that $E$. coli, coliforms, enterococci, and total psychrotrophic aerobic bacterial counts were significantly higher in open well water compared to borehole water [63].

Table 6. Reported correlation between risk management practices and microbial prevalence across studies conducting microbial analysis as a part of the study method $(\mathrm{N}=11)$.

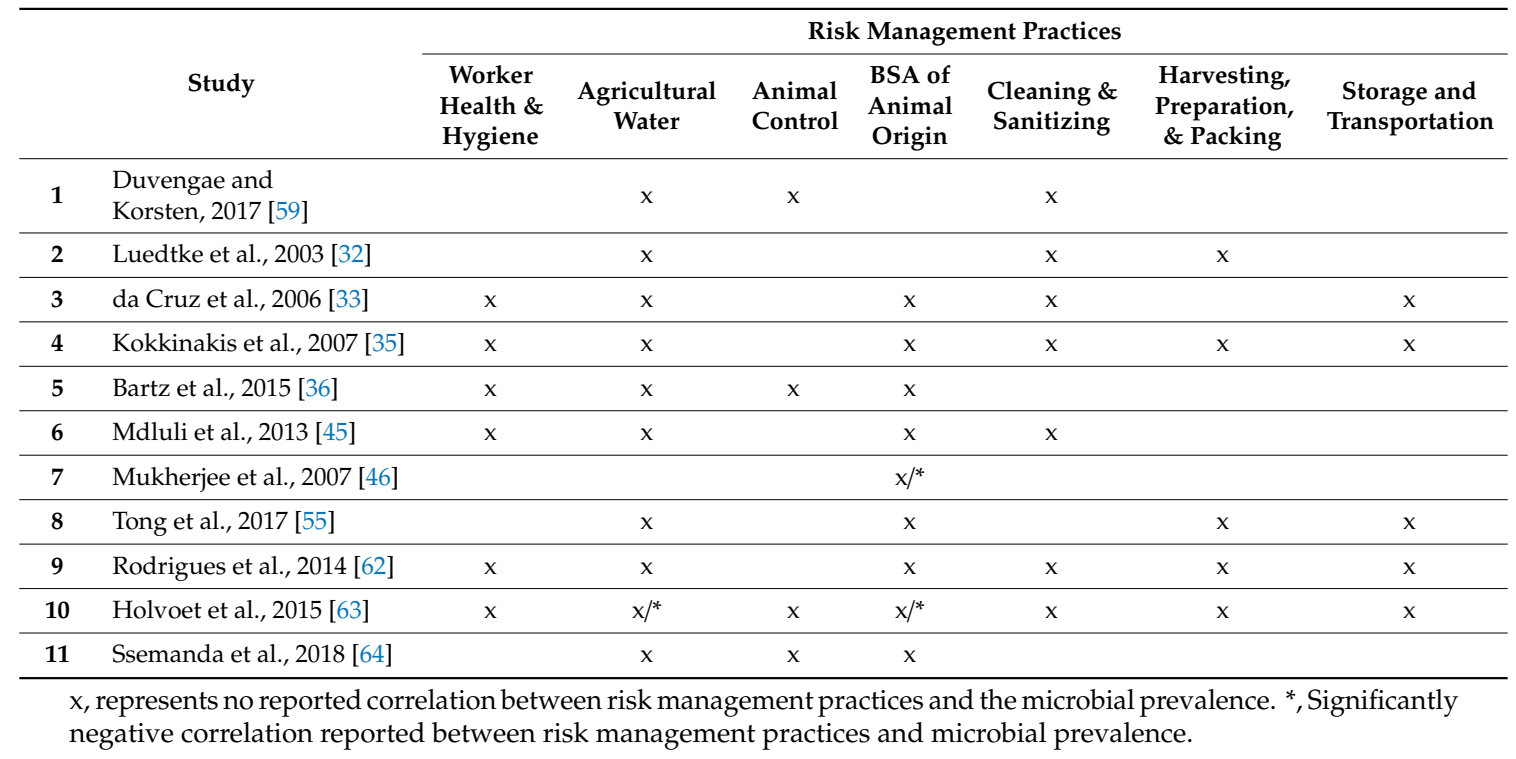

\section{Discussion}

\subsection{Study Characteristics}

Most (26 of 36) studies included in our sample were conducted in developed countries and, thus, the results cannot be easily transferred to developing countries, as resources, regulations, and farming systems are widely different $[66,67]$. Given that developing countries are primary exporters of fruits and vegetables, particularly to developed countries, studies need to be conducted in these countries to help us better understand potential contamination risks for imported product that are associated with environmental characteristics [68-70]. Most investigators $(n=23)$ administered surveys rather than conducted on-farm observations, which allows one to collect data from a large sample size over a large geographic region. The disadvantage though is that surveys are dependent on recall vs. real-time observations, possibly making the results less reliable. On-farm observations were not commonly performed, presumably because they are more expensive and time consuming to conduct [71-73]. Moreover, the sample size is typically smaller than in surveys, and the results are not generalizable to 
the target population of interest [74]. On-farm observations in conjunction with surveys could provide a more comprehensive data set to understand potential risk factors on produce farms.

\subsection{Quality Assessment}

The scores were low for all quality criteria, except for the validation of the instrument/tool, suggesting future studies need to be more rigorously designed and executed. For example, many studies in our sample did not report powering their sample size [74]. In addition, most studies did not report training the data collectors on implementation of the study protocol, which could result in inconsistency among data collectors, hence unreliable data sets [72]. Lastly, the data sets were not cleaned or checked for accuracy before analysis, which could lead to poor analyses and improper conclusions [75]. It is important to note that these results were based on what the authors reported in the paper so these tasks might have been performed.

\subsection{Environmental Characteristics}

Characteristics of agricultural water were commonly assessed, presumably because of how important water is in agricultural production as well as the number of produce-associated outbreaks attributed to contaminated water [14,76-78]. The use of high-risk water sources, the lack of water treatment, and the use of unsafe water application methods were all reported, illustrating that there are many opportunities to contaminate produce during growing activities [79-82]. Possible reasons for using unsafe sources could be the cost associated with water treatment, grower's lack of concern about source water quality, and grower perceptions about water quality [83]. Although farms have toilets and handwashing stations, these sanitary facilities were often not located at a convenient distance, making it difficult to follow hygienic practices $[14,84-86]$. Renting toilets and handwashing stations, providing clean water and handwashing materials, and maintaining those facilities can be expensive $[84,87,88]$. This may lead to purchasing an inadequate number of toilets and handwashing stations for workers, possibly explaining why these facilities were not conveniently located.

Some farms used untreated BSA of animal origin. To produce pathogen-free composted manure, growers need additional money, time, physical resources, and knowledge regarding proper composting $[14,89,90]$. Therefore, farms that do not have adequate resources to compost manure properly might use raw manure [89]. Only one study assessed the types of BSA of animal origin storage [48]. Most farms in Pires et al. (2018) study stored BSA of animal origin in piles with a lack of protective measures [48], such as covers, berms, and fences, which are needed to reduce cross-contamination from BSA of animal origin piles [14,91,92].

In four studies, growers reported using different types of cleaning and sanitization material. Of those, some farms only used water for cleaning and sanitization of equipment and tools. This practice could be due to lack of awareness by growers about proper cleaning and sanitizing to reduce pathogen levels, the inability to afford suitable cleaning and sanitizing products, or the unavailability of proper cleaning and sanitization agents [21]. In addition, growers' lack of concern regarding cleaning and sanitization could also be the cause [83].

Animal control was the least assessed factor. The most common animal deterrent used was fences. Although the initial cost for fences is high, farmers may use fences because of their effectiveness and the long-term advantages in reducing recurring damages [93,94]. One of the issues reported regarding animal control was wild and/or domesticated animal access to production fields and water sources [95]. This could be due to the lack of availability of deterrents that require additional money and maintenance [94], ineffectiveness of preventive measures used [83], and deliberately or unintentionally allowing of livestock or pets to the production areas without the knowledge that animal feces can spread human pathogens. 


\subsection{Relationship between Environmental Characteristics and RMP}

Only one study tested the relationship between environmental characteristics and RMP [34]. This study revealed that availability of handwashing materials significantly increased the frequency of handwashing by workers. Most workers are only paid for their designated tasks; therefore, workers may not be willing to spend additional time on sanitary practices [86]. Providing adequate resources may reduce the barriers and motivate workers to follow proper hygienic practices [86,96]. However, further studies need to be carried out to determine the relationship between other environmental characteristics and RMP.

\subsection{Relationship between RMP and Microbial Prevalence}

Not surprisingly, the use of cattle manure significantly increased the risk of E. coli contamination, most likely because ruminants, such as cattle, naturally have $E$. coli, including pathogenic, Shiga toxinproducing E. coli such as O157:H7 in their intestines; thus, they often shed higher numbers of pathogenic E. coli than other animals [89,97-99]. In addition to BSA of animal origin, microbial prevalence was significantly higher in open well water rather than borehole water, presumably, because borehole water is better protected from external contamination sources than open well water [100]. The disadvantage is that construction of borehole wells requires additional money and specialized equipment, which may not be affordable to farms with limited resources.

\section{Limitations}

This review was conducted using eight databases and search engines that were available through the Clemson University library system. Other relevant databases and search engines might be available. In addition, we only reviewed articles published in English, so we might have excluded relevant articles written in other languages. Lastly, because some studies did not clearly differentiate between the environmental characteristics and RMP available on the farm, it was sometimes difficult to distinguish these from the RMP.

\section{Conclusions}

Our review highlighted the physical resources available to implement RMP on produce farms based on 36 individual studies. Although some farms had the resources to implement RMP, such as sanitary facilities and handwashing aids, other farms did not, which adversely impacts these farms' ability to implement RMP. It is presumed that the cost of implementing RMP is a key factor. Thus, educational interventions need to address how to use existing or lower cost resources as opposed to requiring additional monetary inputs. Ease in implementing RMP and grower perceptions about the importance of RMP might also influence resource availability. Future studies should aim to identify grower perceptions and barriers to implementation of RMP. Lastly, the resources need to implement RMP variably according to the type of produce grown as well as the production system. Therefore, produce specific on-farm environmental assessments will be valuable for identifying resources needed for RMP implementation. Such findings can be used to inform the development of interventions that are commodity-specific.

Most studies in our sample reported the presence/absence and/or the conditions of the environmental characteristics with only one study testing the relationship between the environmental characteristics and RMP. This illustrates the need to determine these relationships between environmental characteristics and implementation of RMP. In addition, more studies should be conducted in developing countries given the volume of produce imports into developed countries such as the United States [101]. The quality assessment of the studies suggests that future work should be more rigorously designed and executed as well as properly reported to provide better quality evidence. 
Author Contributions: Conceptualization, D.N.J., T.A.Y., L.T.T.C., A.M.F., and K.E.G.; Methodology, D.N.J. and A.M.F.; Validation, D.N.J. and A.M.F.; Formal Analysis, D.N.J. and L.T.T.C.; Investigation, D.N.J. and L.T.T.C.; Resources, A.M.F.; Data Curation, D.N.J. and L.T.T.C.; Writing-Original Draft Preparation, D.N.J., L.T.T.C., and A.M.F.; Writing-Review and Editing, D.N.J., L.T.T.C., T.A.Y., A.M.F., and K.E.G.; Visualization, D.N.J.; Supervision, A.M.F.; Project Administration, A.M.F. and K.E.G.; Funding Acquisition, A.M.F. and K.E.G. All authors have read and agreed to the published version of the manuscript.

Funding: This work was funded by the National Institute of Food and Agriculture (NIFA), U.S. Department of Agriculture (USDA), under award number 2017-70020-27255.

Conflicts of Interest: The authors declare no conflict of interest.

\section{References}

1. FAO. FAOSTAT. Food Supply-Crops Primary Equivalent. 2019. Available online: http://www.fao.org/ faostat/en/\#data/CC/ (accessed on 12 October 2020).

2. Carstens, C.K.; Salazar, J.K.; Darkoh, C. Multistate outbreaks of foodborne illness in the United States associated with fresh produce from 2010-2017. Front. Microbiol. 2019, 10, 2667. [CrossRef] [PubMed]

3. Painter, J.A.; Hoekstra, R.M.; Ayers, T.; Tauxe, R.V.; Braden, C.R.; Angulo, F.J.; Griffin, P.M. Attribution of foodborne illnesses, hospitalizations, and deaths to food commodities by using outbreak data, United States, 1998-2008. Emerg. Infect. Dis. 2013, 19, 407-415. [CrossRef] [PubMed]

4. Bennett, S.D.; Sodha, S.V.; Ayers, T.L.; Lynch, M.F.; Gould, L.H.; Tauxe, R.V. Produce-associated foodborne disease outbreaks, USA, 1998-2013. Epidemiol. Infect. 2018, 146, 1397-1406. [CrossRef]

5. Callejón, R.M.; Rodríguez-Naranjo, M.I.; Ubeda, C.; Hornedo-Ortega, R.; Garcia-Parrilla, M.C.; Troncoso, A.M. Reported foodborne outbreaks due to fresh produce in the United States and European Union: Trends and causes. Foodborne Pathog. Dis. 2015, 12, 32-38. [CrossRef] [PubMed]

6. Bélanger, P.; Tanguay, F.; Hamel, M.; Phypers, M. Foodborne illness: An overview of foodborne outbreaks in Canada reported through outbreak summaries: 2008-2014. Can. Commun. Dis. Rep. 2015, 41, 254. [CrossRef]

7. Bartz, F.E.; Lickness, J.S.; Heredia, N.; De Aceituno, A.F.; Newman, K.L.; Hodge, D.W.; Jaykus, L.-A. Contamination of fresh produce by microbial indicators on farms and in packing facilities: Elucidation of environmental routes. Appl. Environ. Microbiol. 2017, 83, e02984-16. [CrossRef]

8. CDC. How Food Gets Contaminated-The Food Production Chain. 2017. Available online: https://www.cdc. gov/foodsafety/production-chain.html/ (accessed on 29 January 2020).

9. FDA. FSMA Final Rule on Produce Safety. 2019. Available online: https://www.fda.gov/food/food-safetymodernization-act-fsma/fsma-final-rule-produce-safety/ (accessed on 11 October 2019).

10. GLOBALGAP. 2020. Available online: https://www.globalgap.org/uk_en/ (accessed on 20 January 2020).

11. FDA. Guidance for Industry: Guide to Minimize Microbial Food Safety Hazards for Fresh Fruits and Vegetables. 1998. Available online: https://www.fda.gov/regulatory-information/search-fda-guidance-documents/ guidance-industry-guide-minimize-microbial-food-safety-hazards-fresh-fruits-and-vegetables (accessed on 15 May 2020).

12. CANADAGAP. 2020. Available online: https://www.canadagap.ca/ (accessed on 15 February 2020).

13. FDA. Standards for the Growing, Harvesting, Packing, and Holding of Produce for Human Consumption. 2015. Available online: https://www.federalregister.gov/documents/2015/11/27/2015-28159/standards-for-thegrowing-harvesting-packing-and-holding-of-produce-for-human-consumption (accessed on 5 June 2020).

14. Produce Safety Alliance. PSA Curriculum. 2020. Available online: https://producesafetyalliance.cornell.edu/ curriculum/ (accessed on 11 January 2020).

15. Laury-Shaw, A.; Strohbehn, C.; Naeve, L.; Wilson, L.; Domoto, P. Current trends in food safety practices for small-scale growers in the Midwest. Food Prot. Trends 2015, 35, 461-469.

16. Fraser, A.M.; Simmons, O.D. Food safety education: Training farm workers in the US fresh produce sector. In Sustainability Challenges in the Agrofood Sector; Bhat, R., Ed.; John Wiley \& Sons Ltd.: Hoboken, NJ, USA, 2017; pp. 643-659.

17. Mahmoud, B.S.; Stafne, E.T.; Coker, C.H.; Bachman, G.R.; Bell, N. Implementing good practices programs to encourage production of high-quality, safer produce in Mississippi. J. Ext. 2016, 54, n4. 
18. Shaw, A.M.; Strohbehn, C.H.; Naeve, L.L.; Domoto, P.A.; Wilson, L.A. Knowledge gained from Good Agricultural Practices courses for Iowa growers. J. Ext. 2015, 53, 5RIB3.

19. Nayak, R.; Tobin, D.; Thomson, J.; Radhakrishna, R.B.; Laborde, L.F. Evaluation of on-farm food safety programming in Pennsylvania: Implications for extension. J. Ext. 2015, 53, 1FEA9.

20. Kline, T.R.; Kneen, H.; Barrett, E.; Kleinschmidt, A.; Doohan, D. Adapting extension food safety programming for vegetable growers to accommodate differences in ethnicity, farming scale, and other individual factors. J. Ext. 2012, 50, 1-4.

21. Clements, D.P.; Bihn, E.A. The Impact of Food Safety Training on the Adoption of Good Agricultural Practices on Farms. In Safety and Practice for Organic Food; Biswas, D., Micallef, S.A., Eds.; Elsevier BV: Amsterdam, The Netherlands, 2019; pp. 321-344.

22. Bihn, E.A.; Springer, L.; Pineda-Bermúdez, L. Local Food Safety Collaborative Need Assessment Report; Department of Food Science, Cornell University: Ithaca, NY, USA, 2015.

23. Lichtenberg, E.; Page, E.T. Prevalence and cost of on-farm produce safety measures in the Mid-Atlantic. Food Control 2016, 69, 315-323. [CrossRef]

24. Bovay, J.; Ferrier, P.; Zhen, C. Estimated Costs for Fruit and Vegetable Producers to Comply with the Food Safety Modernization Act's Produce Rule; U.S. Department of Agriculture, Economic Research Service: Washington, DC, USA, 2018; p. 195.

25. Calder, L.; Simmons, G.; Thornley, C.; Taylor, P.; Pritchard, K.; Greening, G.; Bishop, J. An outbreak of hepatitis A associated with consumption of raw blueberries. Epidemiol. Infect. 2003, 131, 745-751. [CrossRef]

26. Gelting, R.J.; Baloch, M. A systems analysis of irrigation water quality in environmental assessments related to foodborne outbreaks. Aquat. Procedia 2013, 2, 130-137. [CrossRef]

27. Buchholz, U.; Bernard, H.; Werber, D.; Böhmer, M.M.; Remschmidt, C.; Wilking, H.; Deleré, Y.; Der Heiden, M.A.; Adlhoch, C.; Dreesman, J.; et al. German outbreak of Escherichia coli O104: H4 associated with sprouts. N. Engl. J. Med. 2011, 365, 1763-1770. [CrossRef] [PubMed]

28. Gelting, R.J.; Baloch, M.A.; Zarate-Bermudez, M.A.; Selman, C. Irrigation water issues potentially related to the 2006 multistate E. coli O157:H7 outbreak associated with spinach. Agric. Water Manag. 2011, 98, 1395-1402. [CrossRef]

29. Mccollum, J.T.; Cronquist, A.B.; Silk, B.J.; Jackson, K.; O'Connor, K.A.; Cosgrove, S.; Gossack, J.P.; Parachini, S.S.; Jain, N.S.; Ettestad, P.; et al. Multistate outbreak of listeriosis associated with cantaloupe. N. Engl. J. Med. 2013, 369, 944-953. [CrossRef] [PubMed]

30. Greene, S.K.; Daly, E.R.; Talbot, E.A.; Demma, L.J.; Holzbauer, S.; Patel, N.J.; Hill, T.A.; Walderhaug, M.O.; Hoekstra, R.M.; Lynch, M.F.; et al. Recurrent multistate outbreak of Salmonella Newport associated with tomatoes from contaminated fields, 2005. Epidemiol. Infect. 2008, 136, 157-165. [CrossRef]

31. Faour-Klingbeil, D.; Murtada, M.; Kuri, V.; Todd, E.C.D. Understanding the routes of contamination of ready-to-eat vegetables in the Middle East. Food Control 2016, 62, 125-133. [CrossRef]

32. Luedtke, A.N.; Chapman, B.; Powell, D.M. Implementation and analysis of an on-farm food safety program for the production of greenhouse vegetables. J. Food Prot. 2003, 66, 485-489. [CrossRef]

33. Da Cruz, A.G.; Cenci, S.A.; Maia, M.C.A. Good agricultural practices in a Brazilian produce plant. Food Control 2006, 17, 781-788. [CrossRef]

34. Pate, M.L.; Nummer, B. Hand safety for specialty crop production workers: A pilot study investigating frequencies of minor open-wound hand injuries and presence of pathogenic bacteria. J. Agric. Saf. Health 2013, 19, 227-236. [PubMed]

35. Kokkinakis, E.; Boskou, G.; Fragkiadakis, G.A.; Kokkinaki, A.; Lapidakis, N. Microbiological quality of tomatoes and peppers produced under the good agricultural practices protocol AGRO 2-1 \& 2-2 in Crete, Greece. Food Control 2007, 18, 1538-1546. [CrossRef]

36. Bartz, S.; Hessel, C.T.; Rodrigues, R.D.Q.; Possamai, A.; Perini, F.O.; Jacxsens, L.; Uyttendaele, M.; Bender, R.J.; Tondo, E.C. Insights in agricultural practices and management systems linked to microbiological contamination of lettuce in conventional production systems in Southern Brazil. Int. J. Food Contam. 2015, 2, 7. [CrossRef]

37. Öner, G.; Ișin, Ș. Evaluation of fruit-growers' practices in terms of GLOBALGAP criteria in Aegean region, Turkey. Ege Üniv. Ziraat Fak. Derg. 2013, 1, 305-314. 
38. Ganpat, W.; Badrie, N.; Walter, S.; Roberts, L.; Nandlal, J.; Smith, N. Compliance with Good Agricultural Practices (GAPs) by state-registered and non-registered vegetable farmers in Trinidad, West Indies. Food Secur. 2014, 6, 61-69. [CrossRef]

39. Adalja, A.; Lichtenberg, E. Implementation challenges of the food safety modernization act: Evidence from a national survey of produce growers. Food Control 2018, 89, 62-71. [CrossRef]

40. Van Asseldonk, M.A.P.M.; Malaguti, L.; Breukers, M.L.H.; Van Der Fels-Klerx, H.J. Understanding preferences for interventions to reduce microbiological contamination in Dutch vegetable production. J. Food Prot. 2018, 81, 892-897. [CrossRef]

41. Bihn, E.A.; Smart, C.D.; Hoepting, C.A.; Worobo, R.W. Use of surface water in the production of fresh fruits and vegetables: A survey of fresh produce growers and their water management practices. Food Prot. Trends 2013, 33, 307-314.

42. Dzingirayi, G.; Korsten, L. Assessment of primary production of horticultural safety management systems of mushroom farms in South Africa. J. Food Prot. 2016, 79, 1188-1196. [CrossRef]

43. Harrison, J.A.; Gaskin, J.W.; Harrison, M.A.; Cannon, J.L.; Boyer, R.R.; Zehnder, G.W. Survey of food safety practices on small to medium-sized farms and in farmers markets. J. Food Prot. 2013, 76, 1989-1993. [CrossRef]

44. Ivey, M.L.L.; Lejeune, J.T.; Miller, S. Vegetable producers' perceptions of food safety hazards in the midwestern USA. Food Control 2012, 26, 453-465. [CrossRef]

45. Mdluli, F.; Thamaga-Chitja, J.; Schmidt, S. Appraisal of hygiene indicators and farming practices in the production of leafy vegetables by organic small-scale farmers in uMbumbulu (Rural KwaZulu-Natal, South Africa). Int. J. Environ. Res. Public Health 2013, 10, 4323-4338. [CrossRef] [PubMed]

46. Mukherjee, A.; Speh, D.; Diez-Gonzalez, F. Association of farm management practices with risk of Escherichia coli contamination in pre-harvest produce grown in Minnesota and Wisconsin. Int. J. Food Microbiol. 2007, 120, 296-302. [CrossRef] [PubMed]

47. Park, S.; Navratil, S.; Gregory, A.; Bauer, A.; Srinath, I.; Szonyi, B.; Nightingale, K.; Anciso, J.; Jun, M.; Han, D.; et al. Farm management, environment, and weather factors jointly affect the probability of spinach contamination by generic Escherichia coli at the preharvest stage. Appl. Environ. Microbiol. 2014, 80, 2504-2515. [CrossRef]

48. Pires, A.F.A.; Millner, P.D.; Baron, J.; Jay-Russell, M.T. Assessment of current practices of organic farmers regarding biological soil amendments of animal origin in a multi-regional US study. Food Prot. Trends 2018, 38, 347-362.

49. Soon, J.M. Food safety perceptions and practices of selected UK fresh produce farms. Qual. Assur. Saf. Crop. Foods 2012, 4, 61-76. [CrossRef]

50. Cohen, N.; Hollingsworth, C.S.; Olson, R.B.; Laus, M.J.; Coli, W.M. Farm food safety practices: A survey of New England growers. Food Prot. Trends 2005, 25, 363-370.

51. Hultberg, A.; Schermann, M.; Tong, C. Results from a mail survey to assess Minnesota vegetable growers' adherence to Good Agricultural Practices. HortTechnology 2012, 22, 83-88. [CrossRef]

52. Marine, S.C.; Martin, D.A.; Adalja, A.; Mathew, S.; Everts, K.L. Effect of market channel, farm scale, and years in production on mid-Atlantic vegetable producers' knowledge and implementation of Good Agricultural Practices. Food Control 2016, 59, 128-138. [CrossRef]

53. Rangarajan, A.; Pritts, M.P.; Reiners, S.; Pedersen, L.H. Focusing food safety training based on current grower practices and farm scale. HortTechnology 2002, 12, 126-131. [CrossRef]

54. Sinkel, D.; Khouryieh, H.; Daday, J.K.; Stone, M.; Shen, C. Knowledge and implementation of Good Agricultural Practices among Kentucky fresh produce farmers. Food Prot. Trends 2018, 38, 111-121.

55. Tong, C.; Schermann, M.; Diez-Gonzalez, F.; Rossbach, J. Food safety risks of leafy greens from small-acreage farms in Minnesota. J. Ext. 2017, 55, 4RIB4.

56. Jackson, C.; Larcher, D.; Goodrich-Schneider, R.; Gravani, R.B.; Bihn, E.A.; Schneider, K.R. Determining the effect of Good Agricultural Practices awareness on implementation: A multi-state survey. Food Prot. Trends 2007, 27, 684-693.

57. Baur, P.; Driscoll, L.; Gennet, S.; Karp, D. Inconsistent food safety pressures complicate environmental conservation for California produce growers. Calif. Agric. 2016, 70, 142-151. [CrossRef] 
58. Beretti, M.; Stuart, D. Food safety and environmental quality impose conflicting demands on Central Coast growers. Calif. Agric. 2008, 62, 68-73. [CrossRef]

59. Duvenage, S.; Korsten, L. Assessment of foodborne pathogen presence in the peach supply chain and its potential risk to the end consumer. Food Control 2017, 78, 374-382. [CrossRef]

60. Hamilton, K.E.; Umber, J.; Hultberg, A.; Tong, C.; Schermann, M.; Diez-Gonzalez, F.; Bender, J.B. Validation of Good Agricultural Practices (GAP) on Minnesota vegetable farms. Foodborne Pathog. Dis. 2015, 12, 145-150. [CrossRef]

61. Ellis, J.D.; Strohbehn, C.H.; Henroid, D.H., Jr. Assessing on-farm food handling practices of Iowa-grown produce and eggs in regard to food safety. Food Prot. Trends 2005, 25, 758-761.

62. Rodrigues, R.D.Q.; Loiko, M.R.; de Paula, C.M.D.; Hessel, C.T.; Jacxsens, L.; Uyttendaele, M.; Bender, R.J.; Tondo, E.C. Microbiological contamination linked to implementation of good agricultural practices in the production of organic lettuce in Southern Brazil. Food Control 2014, 42, 152-164. [CrossRef]

63. Holvoet, K.; Sampers, I.; Seynnaeve, M.; Jacxsens, L.; Uyttendaele, M. Agricultural and management practices and bacterial contamination in greenhouse versus open field lettuce production. Int. J. Environ. Res. Public Health 2014, 12, 32-63. [CrossRef]

64. Ssemanda, J.N.; Reij, M.W.; Van Middendorp, G.; Bouw, E.; Van Der Plaats, R.; Franz, E.; Muvunyi, C.M.; Bagabe, M.C.; Zwietering, M.H.; Joosten, H. Foodborne pathogens and their risk exposure factors associated with farm vegetables in Rwanda. Food Control 2018, 89, 86-96. [CrossRef]

65. USDOL (United States Department of Labor). Fact Sheet \#51: Field Sanitation Standards under the Occupational Safety and Health Act. Available online: https://www.dol.gov/sites/dolgov/files/WHD/legacy/ files/whdfs51.pdf/ (accessed on 8 July 2020).

66. Grace, D. Food safety in developing countries: Research gaps and opportunities. In White Paper; International Livestock Research Institute: Nairobi, Kenya, 2017.

67. Oloo, B.; Daisy, L.; Oniang'O, R. Food safety legislation in some developing countries. In Food Safety-Some Global Trends; El-Samragy, Y., Ed.; IntechOpen: London, UK, 2018; pp. 19-35.

68. Frankowska, A.; Jeswani, H.K.; Azapagic, A. Environmental impacts of vegetables consumption in the UK. Sci. Total. Environ. 2019, 682, 80-105. [CrossRef] [PubMed]

69. Denis, N.; Zhang, H.; Leroux, A.; Trudel, R.; Bietlot, H. Prevalence and trends of bacterial contamination in fresh fruits and vegetables sold at retail in Canada. Food Control 2016, 67, 225-234. [CrossRef]

70. Johnson, R. The U.S. Trade Situation for Fruit and Vegetable Products; Congressional Research Services: Washington, DC, USA, 2016.

71. Driscoll, D.L. Introduction to primary research: Observations, surveys, and interviews. In Writing Spaces: Readings on Writings; Lowe, C., Zemliansky, P., Eds.; Parlor Press: Anderson, SC, USA, 2011; Volume 2, pp. 153-174.

72. Phellas, C.; Bloch, A.; Seale, C. Structured methods: Interviews, questionnaires and observations. In Researching Society and Culture; Seale, C., Ed.; SAGE Publications: Thousand Oaks, CA, USA, 2011; pp. 181-205.

73. Muijs, D. Designing Non-Experimental Studies. Doing Quantitative Research in Education with SPSS; SAGE Publications: Thousand Oaks, CA, USA, 2010.

74. Kadam, P.; Bhalerao, S. Sample size calculation. Int. J. Ayurveda Res. 2010, 1, 55. [PubMed]

75. Salkind, N. Encyclopedia of Research Design; SAGE Publications: Thousand Oaks, CA, USA, 2010.

76. CDC. Foodborne Outbreaks. List of Selected Multistate Foodborne Outbreak Investigations. 2020. Available online: https://www.cdc.gov/foodsafety/outbreaks/multistate-outbreaks/outbreaks-list.html/ (accessed on 15 February 2020).

77. Jay-Russell, M.; Cooley, M.; Carychao, D.; Wiscomb, G.W.; Sweitzer, R.A.; Crawford-Miksza, L.; Farrar, J.A.; Lau, D.K.; O'Connell, J.; Millington, A.; et al. Escherichia coli O157: H7 in feral swine near spinach fields and cattle, central California coast. Emerg. Infect. Dis. 2007, 13, 1908-1911. [CrossRef]

78. Soderstrom, A.; Österberg, P.; Lindqvist, A.; Jönsson, B.; Lindberg, A.; Ulander, S.B.; Welinder-Olsson, C.; Löfdahl, S.; Kaijser, B.; De Jong, B.; et al. A large Escherichia coli O157 outbreak in Sweden associated with locally produced lettuce. Foodborne Pathog. Dis. 2008, 5, 339-349. [CrossRef]

79. Castro-Rosas, J.; Cerna-Cortés, J.F.; Méndez-Reyes, E.; Lopez-Hernandez, D.; Gómez-Aldapa, C.A.; Estrada-García, T. Presence of faecal coliforms, Escherichia coli and diarrheagenic E. coli pathotypes in 
ready-to-eat salads, from an area where crops are irrigated with untreated sewage water. Int. J. Food Microbiol. 2012, 156, 176-180. [CrossRef]

80. Ensink, J.H.J.; Mahmood, T.; Dalsgaard, A. Wastewater-irrigated vegetables: Market handling versus irrigation water quality. Trop. Med. Int. Health 2007, 12, 2-7. [CrossRef]

81. Karg, H.; Drechsel, P. Motivating behaviour change to reduce pathogenic risk where unsafe water is used for irrigation. Water Int. 2011, 36, 476-490. [CrossRef]

82. Steele, M.; Odumeru, J. Irrigation water as source of foodborne pathogens on fruit and vegetables. J. Food Prot. 2004, 67, 2839-2849. [CrossRef] [PubMed]

83. Parker, J.; Wilson, R.; Lejeune, J.T.; Doohan, D.J. Including growers in the "food safety" conversation: Enhancing the design and implementation of food safety programming based on farm and marketing needs of fresh fruit and vegetable producers. Agric. Hum. Values 2012, 29, 303-319. [CrossRef]

84. Harrison, J.A. Food safety hazards identified on small farms. In Food Safety for Farmers Markets: A Guide to Enhancing Safety of Local Foods; Harrison, J.A., Ed.; Springer: Cham, Switzerland, 2017; pp. 13-21.

85. Rangarajan, A.; Bihn, E.A.; Gravani, R.B.; Scott, D.L.; Pritts, M.P. Food Safety Begins on the Farm: A Grower's Guide; Cornell University: Ithaca, NY, USA, 2000.

86. Soon, J.; Baines, R. Food safety training and evaluation of handwashing intention among fresh produce farm workers. Food Control 2012, 23, 437-448. [CrossRef]

87. Volk, J. Compact Farms: 15 Proven Plans for Market Farms on 5 Acres or Less; Includes Detailed Farm Layouts for Productivity and Efficiency; Burns, D., Ed.; Storey Publishing: North Adams, MA, USA, 2017.

88. Woods, M.; Thornsbury, S. Costs of Adopting Good Agricultural Practices (GAPs) to Ensure Food Safety in Fresh Strawberries; Department of Agricultural Economics, Michigan State University: East Lansing, MI, USA, 2005.

89. FDA. Raw Manure under the FSMA Final Rule on Produce Safety. 2018. Available online: https://www.fda. gov/food/food-safety-modernization-act-fsma/raw-manure-under-fsma-final-rule-produce-safety/ (accessed on 5 June 2020).

90. Wander, M. Managing Manure Fertilizers in Organic Systems. eOrganic. 2015. Available online: https: //eorganic.org/node/3132 (accessed on 8 July 2020).

91. Suslow, T.V. Key Points of Control and Management of Microbial Food Safety: Information for Producers, Processors, and Handlers of Fresh Market Tomatoes; Agriculture and Natural Resources, University of California: Oakland, CA, USA, 2004.

92. Brandl, M.T. Fitness of human enteric pathogens on plants and implications for food safety. Annu. Rev. Phytopathol. 2006, 44, 367-392. [CrossRef] [PubMed]

93. VerCauteren, K.C.; Lavelle, M.J.; Hygnstrom, S. From the field: Fences and deer-damage management: A review of designs and efficacy. Wildl. Soc. Bull. 2006, 34, 191-200. [CrossRef]

94. Rice, D.H. Produce Contamination by Other Wildlife. In The Produce Contamination Problem; Elsevier BV: Amsterdam, The Netherlands, 2014; pp. 167-183.

95. DeCol, L.T.; Casarin, L.S.; Hessel, C.T.; Batista, A.C.F.; Allende, A.; Tondo, E.C. Microbial quality of irrigation water used in leafy green production in Southern Brazil and its relationship with produce safety. Food Microbiol. 2017, 65, 105-113. [CrossRef]

96. Pilling, V.K.; Brannon, L.A.; Shanklin, C.W.; Howells, A.D.; Roberts, K.R. Identifying specific beliefs to target to improve restaurant employees' intentions for performing three important food safety behaviors. J. Am. Diet. Assoc. 2008, 108, 991-997. [CrossRef]

97. Grauke, L.J.; Kudva, I.T.; Yoon, J.W.; Hunt, C.W.; Williams, C.J.; Hovde, C.J. Gastrointestinal tract location of Escherichia coli O157: H7 in ruminants. Appl. Environ. Microbiol. 2002, 68, 2269-2277. [CrossRef]

98. Berry, E.D.; Millner, P.D.; Wells, J.E.; Kalchayanand, N.; Guerini, M.N. Fate of naturally occurring Escherichia coli O157: H7 and other zoonotic pathogens during minimally managed bovine feedlot manure composting processes. J. Food Prot. 2013, 76, 1308-1321. [CrossRef]

99. Tabe, N.N.; Rahman, S.; Tabe, E.S.; Doetkott, D.; Ekiri, A.B.; Khaitsa, M.L. Prevalence of Escherichia coli and Salmonella in runoff of two cattle feedlots in North Dakota. Food Prot. Trends 2016, 36, 33-42.

100. Gwimbi, P. The microbial quality of drinking water in Manonyane community: Maseru District (Lesotho). Afr. Health Sci. 2011, 11, 474-480. [PubMed] 
101. USDA ERS. Summary Data on Annual Food Imports, Values and Volumes by Food Category and Source Country, 1999-2017. 2018. Available online: https://www.ers.usda.gov/data-products/us-food-imports/ (accessed on 18 November 2020).

Publisher's Note: MDPI stays neutral with regard to jurisdictional claims in published maps and institutional affiliations.

(C) 2020 by the authors. Licensee MDPI, Basel, Switzerland. This article is an open access article distributed under the terms and conditions of the Creative Commons Attribution (CC BY) license (http://creativecommons.org/licenses/by/4.0/). 